\title{
Low-level and ultralow-volume hollow waveguide based carbon monoxide sensor
}

\author{
Jia Chen ${ }^{1,2, *}$ Andreas Hangauer, ${ }^{1,2}$ Rainer Strzoda, ${ }^{1}$ Maximilian Fleischer, ${ }^{1}$ and Markus-Christian Amann ${ }^{2}$ \\ ${ }^{1}$ Corporate Research and Technologies, Siemens AG, Otto-Hahn-Ring 6, D-80200 München, Germany \\ ${ }^{2}$ Walter Schottky Institut, Technische Universität München, Am Coulombwall 3, D-85748 Garching, Germany \\ *Corresponding author: jia.chen@wsi.tum.de
}

Received July 20, 2010; accepted August 26, 2010;

posted September 27, 2010 (Doc. ID 129352); published October 21, 2010

\begin{abstract}
We demonstrate an ultralow sample volume optical carbon monoxide sensor with detection sensitivity of 180 parts in $10^{9}(1 \sigma$ at $1 \mathrm{~Hz})$. The utilization of a $2.3 \mu \mathrm{m}$ surface-emitting laser directly coupled to a $3 \mathrm{~m}$ hollow capillary fiber as the gas cell is proven to be a compact, sensitive, and cost-efficient gas sensing concept. By mechanical vibration of the fiber, an absorbance resolution of $10^{-5}$ is achieved, which is comparable to single-reflective (double-pass) cells. An improvement of sensitivity over the conventional single-reflective cell is thus approximately linearly scaled with the enhancement of the optical path length, which is usually more than 1 order of magnitude. () 2010 Optical Society of America

OCIS codes: $280.1545,300.6260,300.1030,280.4788,230.7370,060.2340$.
\end{abstract}

Tunable diode laser absorption spectroscopy (TDLAS) based gas sensors have unique advantages such as the lowest possible cross-sensitivity, long-term stability, and nearly fail-safe operation. TDLAS gas sensors using gas permeable fibers as both the gas "pipe" and optical waveguide are very attractive because fibers greatly extend the optical path length compared to conventional single-reflective (i.e., double-pass) gas cells. This is also possible without extending the sensor dimension, because the fiber can be wound up with diameters in the 10-20 cm range. Compared to multipass cells, where longer optical path lengths are achieved by applying multiple reflections on the mirrors on both ends of the cell, e.g., the Herriott cell [1,2] or White cell [3] the sample volume is reduced by several orders of magnitude for the fiber sensor and the alignment is much simplified.

$\mathrm{CO}$ is a trace gas and affects human health whenever present in ambient air due to its toxicity. Furthermore, it is the most prominent gas to be detected for gas sensor based fire detection [4]. Applying the recently developed $2.3 \mu \mathrm{m}$ vertical-cavity surface-emitting lasers (VCSELs) $[\underline{5}, \underline{6}]$, an absorption line in the first-overtone band of $\mathrm{CO}$ is sampled, which is approximately 160 times stronger (peak absorption coefficient $\alpha_{\text {peak }} \approx 0.43 \mathrm{~cm}^{-1}$ ) than its second overtone band at $1.5 \mu \mathrm{m}\left(\alpha_{\text {peak }} \approx 0.0027 \mathrm{~cm}^{-1}\right)$. Utilizing a $10 \mathrm{~cm}$ single-reflective cell, a sensor resolution of 3 parts in $10^{6}(\mathrm{ppm})(1 \sigma$ at $1 \mathrm{~Hz})$ is already achieved at $2.3 \mu \mathrm{m}$ [7]. Using a $3 \mathrm{~m}$ hollow fiber, a sensitivity improvement of a factor of 30 is expected. Possible applications are reliable fire detection, safety monitoring, and breath analysis.

In this Letter, a hollow glass waveguide is used for the detection of carbon monoxide (Type HWEA7501200, Polymicro Technologies). Light guiding is achieved by reflection on the Ag/AgI coating on the inner surface of the fiber. The fiber was originally developed for high-power light transmission in the mid-infrared (MIR) range where conventional fibers cannot be used. The AgI coating has a thickness of $0.3 \mu \mathrm{m}$ for a minimal loss at a wavelength of $2.94 \mu \mathrm{m}$. The fiber has $1.2 \mathrm{ml}$ volume, a length of $3 \mathrm{~m}$, and an inner diameter of $750 \mu \mathrm{m}[8,9]$. Fibers up to $13 \mathrm{~m}$ in length are commercially available.

In Fig. 1(a) the design of the fiber based CO sensor is shown. Because of the low divergence of the VCSEL emission (FWHM: $10^{\circ}-20^{\circ}$ ), direct in- and outcoupling to the fiber (measured FWHM: $\sim 10^{\circ}$ ) is employed. This enables a robust and miniaturized sensor design, because additional coupling/focusing elements are avoided and possible optical interferences caused by parallel optical surfaces are eliminated. Compared to multipass cells, almost no adjustments have to be made. By using adjacent absorption lines of methane, which is filled in the

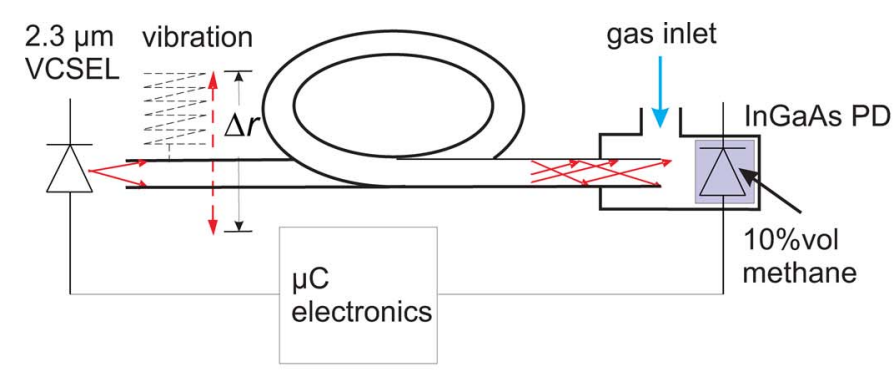

(a)

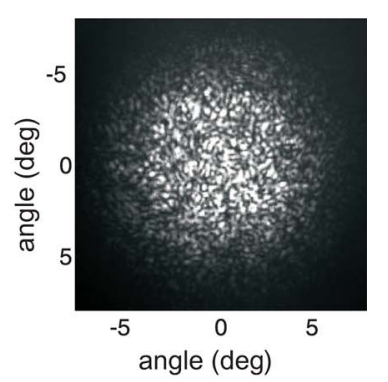

(b)

Fig. 1. (Color online) (a) Experimental setup of the fiber sensor. Mechanical vibration is employed to the fiber at the incoupling end. A reference gas $\left(10 \mathrm{vol} . \% \mathrm{CH}_{4}\right)$ is filled in the photodetector housing to form an integrated reference cell [7]. Compact electronics serve as the sensor control for signal processing and data evaluation. (b) Far field of the fiber: the speckle pattern indicates highly multimode propagation of the light. 
photodetector housing (Fig. 2), the wavelength scale is calibrated inherently without using an external reference cell. In [7] this was introduced for a single-reflective cell. Furthermore, it enables simultaneous tracking of the position of the $\mathrm{CO}$ line and determination of the linear and quadratic current tuning coefficient of the laser.

The sensor resolution in terms of concentration $c_{\text {res }}$ (mixing ratio) is given by

$$
c_{\mathrm{res}}=\frac{a_{\mathrm{res}}}{L \alpha},
$$

where $\alpha$ is the absorption coefficient (unit: $1 / \mathrm{m}$ ), which is an intrinsic property of the gas species. $L$ is the interaction length between light, and $a_{\text {res }}$ (unit: 1 ) is the measurement absorbance resolution, i.e., the smallest detectable fraction of light absorbed by the gas. When the absorption line to be detected is chosen, the concentration resolution $c_{\text {res }}$ is basically given by the ratio between $a_{\text {res }}$ and $L$. Both $a_{\text {res }}$ and $L$ are sensor properties. Some gas/liquid sensing experiments with hollow fibers have been reported so far for MIR sensing using coupling elements such as objectives or off-axis parabolic mirrors [10-12]. However, the empty cell transmission is nonsmooth, which limits $a_{\text {res. }}$. So this could cancel the benefit of a longer optical path length $L$.

In [13] a detailed characterization and analysis of the fiber background are given. The fiber background consists of spectral features caused by the multimode character of the fiber itself. It turned out to be the limiting factor for the sensor resolution in terms of absorbance $a_{\text {res }}$. The multimode light propagation also results in a speckle pattern for the fiber far field [Fig. 1(b)]. It was found that the amplitude of fiber background is inversely proportional to $\sqrt{N}$, with $N$ being the number of speckle points integrated by the photodiode. When vibrating the fiber, the effective number of independent speckle points being collected by the photodiode increases, because the speckle pattern changes over time. According to experiments (Fig. 2), the fiber background has a standard deviation of $8 \times 10^{-5}$ (absorbance) without vibration and $10^{-5}$ with vibration, which is the absorbance resolution achievable with a conventional single-reflective cell [7]. Therefore, the improvement of sensitivity over conven-

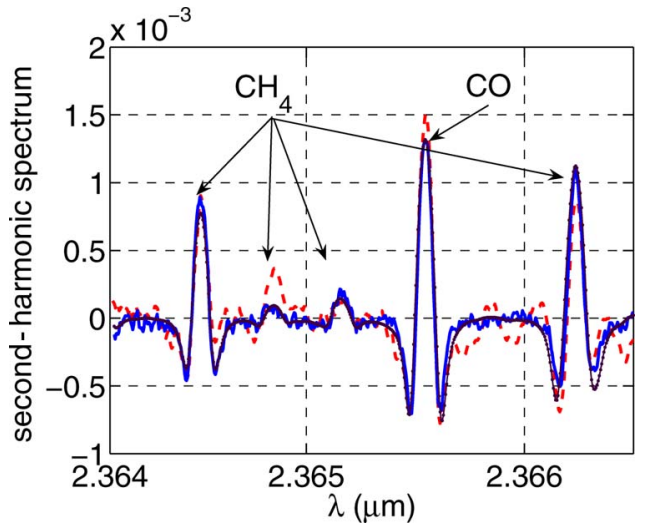

Fig. 2. (Color online) Measured second-harmonic transmission spectrum (from wavelength modulation spectroscopy) of $\mathrm{CO}$ and $\mathrm{CH}_{4}$ with vibration (blue solid curve) and without vibration of the fiber (red dashed curve) and the theoretical spectrum (HITRAN) (black dotted curve). tional cells is expected to be linearly scaled with the enhancement factor of the optical path length [see Eq. (1)].

A $200 \mathrm{~Hz}$ vibration is employed to the fiber end used for incoupling with an optimized vibration amplitude $(\Delta r$ in the several $100 \mu \mathrm{m}$ range). It turned out, practically, that the smoothing effect of the vibration does not depend on the vibration frequency, as long as it is higher than the cutoff frequency of system filters, e.g., an analog low-pass filter and higher than the measurement rate. $200 \mathrm{~Hz}$ is therefore sufficient for a $10 \mathrm{~Hz}$ concentration measurement rate, and it can probably be chosen even lower. The fiber background and sensor baseline are very sensitive to the vibration amplitude, with its influence shown in Fig. 3. If the vibration amplitude is too high (i.e., in the range of the inner diameter of the fiber), the coupled light intensity is fluctuating, too, because of inappropriate coupling conditions, which distort the measurement. If the vibration amplitude is too low, the spectral background is not improved, resulting in a unstable and inaccurate sensor baseline. The Allan plot [14] for the fiber sensor (3 $\mathrm{m}$ fiber length) is shown in Fig. 4. A 180 parts in $10^{9}$ (ppb) concentration resolution $(1 \sigma)$ was obtained for a $1 \mathrm{~s}$ averaging time. For comparison, the sensor with a single-reflective cell (using the same laser and photodiode, $40 \mathrm{~cm}$ optical path length) was also characterized and the expected performance of a $3 \mathrm{~m}$ single-reflective cell is extrapolated by scaling with the path length ratio (Fig. 4). In contrast to the single-reflective cell, a drift of the fiber sensor is indicated by the increase of the Allan standard deviation $\sigma_{\text {Allan }}$ above $100 \mathrm{~s}$. Therefore, the drift must be caused by the fiber itself or the mechanical vibration. The increase at $6 \mathrm{~s}$ in the Allan plot is an artifact of the signal evaluation depending on the sampling scheme and can be improved. The gas was supplied to the fiber with a regulated flow rate realized with a mass flow controller. Overpressures of $<1$ bar already realize gas exchange times significantly below $1 \mathrm{~s}$. If high-speed gas exchange is desired, a certain pressure broadening of the spectral lines may have to be taken into account in the spectral evaluation. The sensor was tested by measuring the exhalation of a smoker (Fig. 5). Cigarette smoke contains a high

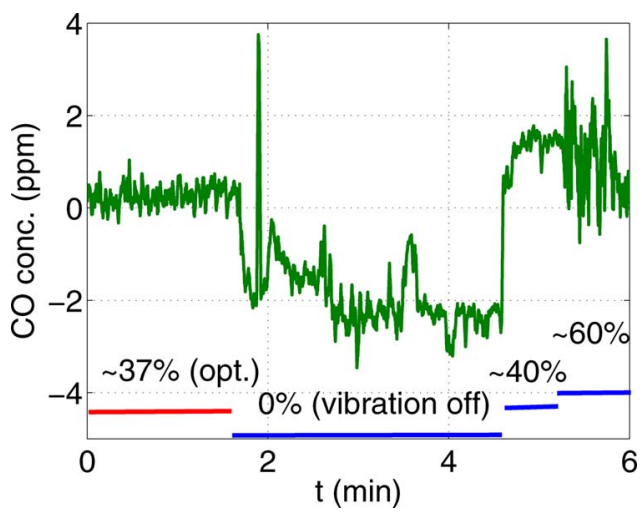

Fig. 3. (Color online) Sensor baseline (output values "without" $\mathrm{CO})$ caused by the fiber spectral background. With optimized vibration amplitude (37\%), the sensor baseline has low noise and almost no offset, whereas other conditions give a strong time-dependent offset $(0 \%$ and $40 \%)$ or high noise $(60 \%)$ on the sensor baseline. The percentage values give the relative vibration strength, because the absolute value is unknown. 


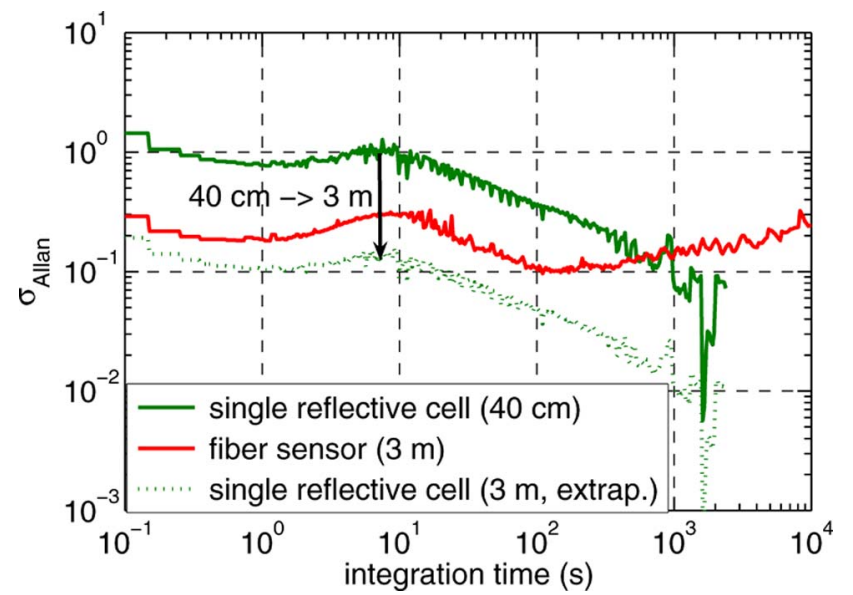

Fig. 4. (Color online) Allan standard deviation [14] versus averaging time shown for the $3 \mathrm{~m}$ fiber based sensor (red solid curve), the $40 \mathrm{~cm}$ single-reflective cell based sensor (green solid curve), and the extrapolated performance of a $3 \mathrm{~m}$ singlereflective based sensor (green dotted curve).

concentration of carbon monoxide, which is very easily taken up by the blood. The affinity of hemoglobin to carbon monoxide is approximately 200 times higher than to oxygen. Exhalation of a smoker was sampled into the fiber, where a CO concentration of $20 \mathrm{ppm}$ is measured. For comparison, the exhalation of a nonsmoker contained a concentration of below $2 \mathrm{ppm}$ in the exhalation. The result is also consistent with another study of over 100,000 people in the European Union [15]: carbon monoxide concentration in exhalation rises by $1-2 \mathrm{ppm}$ per cigarette per day, whereas an average $\mathrm{CO}$ concentration of a nonsmoker is $2-3 \mathrm{ppm}$.

In this Letter, a low-volume and low-level optical carbon monoxide sensor $(1 \sigma: 180 \mathrm{ppb}$ at $1 \mathrm{~Hz})$ was

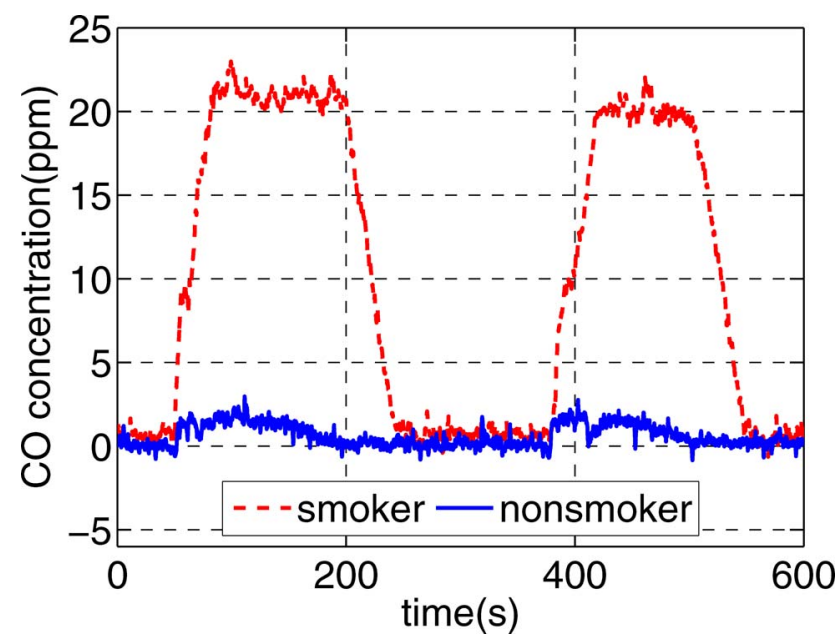

Fig. 5. (Color online) Measured carbon monoxide concentration in exhalation of a smoker (red dashed curve) and a nonsmoker (blue solid curve) in $1 \mathrm{~s}$ time resolution. Each measurement consists of (i) sampling of the exhalation into the fiber, (ii) keeping the gas in the fiber, and (iii) purging with nitrogen. developed. The utilization of a surface-emitting laser directly coupled to a hollow capillary fiber as the gas cell is proven to be a compact, sensitive, and cost-efficient gas sensing concept, which is demonstrated for the first time (to our knowledge).

By applying vibration to the fiber with a moderate frequency of $200 \mathrm{~Hz}$ and a low amplitude of several $100 \mu \mathrm{m}$, the fiber transmission function becomes smooth, so that absorbance on the order of $10^{-5}$ is resolvable with the sensor, which is comparable to conventional cells. Compared to a single-reflective cell based sensor [7], the fiber sensor has a 16-fold improvement in concentration resolution with a 30 times enhancement of the optical path length, while the needed sample volume is simultaneously lowered by several orders of magnitude to $1.3 \mathrm{ml}$. This clearly demonstrates the potential of laser spectroscopic hollow capillary fiber based sensors. An additional severalfold sensitivity improvement is expected by using longer fibers, which is no technical difficulty.

The authors thank VERTILAS GmbH for providing the $2.3 \mu \mathrm{m}$ VCSELs, and the first author gratefully thanks Ralf Meyer for the valuable discussions.

\section{References}

1. D. Herriott, H. Kogelnik, and R. Kompfner, Appl. Opt. 3, 523 (1964).

2. J. B. McManus, P. L. Kebabian, and M. S. Zahniser, Appl. Opt. 34, 3336 (1995).

3. J. U. White, J. Opt. Soc. Am. 32, 285 (1942).

4. A. Hangauer, J. Chen, R. Strzoda, M. Fleischer, and M.-C. Amann, in Conference on Lasers and Electro-Optics (Optical Society of America, 2009), paper CTuA3.

5. M. Ortsiefer, G. Böhm, M. Grau, K. Windhorn, E. Rönneberg, J. Rosskopf, R. Shau, O. Dier, and M.-C. Amann, Electron. Lett. 42, 640 (2006).

6. A. Bachmann, T. Lim, K. Kashani-Shirazi, O. Dier, C. Lauer, and M.-C. Amann, Electron. Lett. 44, 202 (2008).

7. J. Chen, A. Hangauer, R. Strzoda, and M.-C. Amann, "VCSEL-based calibration-free carbon monoxide sensor at $2.3 \mu \mathrm{m}$ with in-line reference cell," Appl. Phys. B (to be published).

8. J. A. Harrington, C. Rabii, and D. Gibson, IEEE J. Sel. Top. Quantum Electron. 5, 948 (1999).

9. R. K. Nubling and J. A. Harrington, Opt. Eng. 37, 2454 (1998).

10. C. Young, S.-S. Kim, Y. Luzinova, M. Weida, D. Arnone, E. Takeuchi, T. Day, and B. Mizaikoff, Sens. Actuators B 140, 24 (2009).

11. S. Hartwig and A. Lambrecht, Technisches Messen 75 , 555 (2008).

12. C. A. Worrell and N. A. Gallen, J. Phys. D 30, 1984 (1997).

13. J. Chen, A. Hangauer, R. Strzoda, and M.-C. Amann, Appl. Opt. 49, 5254 (2010).

14. P. Werle, R. Muecke, and F. Slemr, Appl. Phys. B 57, 131 (1993).

15. B. Dautzenberg, presented at Conference: Communicating Health, the Tobacco Example, Brussels, 9 October 2008 . 